\title{
Trastorno por déficit atencional con hiperactividad en adultos
}

\author{
Arturo Roizblatt $\mathbf{S}^{1}$, Francisco Bustamante $\mathbf{V}^{2 a}$, \\ Félix Bacigalupo I2a. \\ Attention deficit hyperactivity
disorder in adults
}

The attention deficit hyperactivity disorder (ADHD) is the most common diagnosis in child psychiatry, and persists very often in adulthood. These patients have poor lifestyles, especially in affective, work and social areas. Although patients with ADHD have a high rate of comorbidity (e.g. drug and alcohol abuse, depressive and anxiety disorders), this diagnosis is missed by the general physician, deferring its identification and opportune treatment. This article is a review about this disorder and gives tools for its recognition and derivation (Rev Méd Chile 2003; 131: 1195-201).

(Key Words: Anxiety; Attention deficit disorder with hyperactivity; Depression; Hyperkinesis)

Recibido el 3 de enero, 2003. Aceptado en versión corregida el 17 de agosto, 2003.

${ }^{1}$ Departamento de Psiquiatría, Facultad de Medicina, Campus Oriente, Universidad de Chile y ${ }^{2}$ Facultad de Medicina, Universidad de los Andes.

Médicos becarios en Psiquiatría.

$\mathrm{E}^{1}$ Trastorno por Déficit Atencional con Hiperactividad (TDAH) es el diagnóstico psiquiátrico más común de la infancia, con una prevalencia estimada de 3 a $7 \%$, afectando más frecuentemente a los hombres en una proporción de 3 a 9:1 con respecto a las mujeres, aunque es importante destacar, que los síntomas suelen ser más severos en pacientes del sexo femenino ${ }^{1}$. En Chile, Toledo y cols, realizaron un estudio de campo en niños de primer año de enseñanza básica del área occidente de Santiago, encontrando una prevalencia para TDAH de $6,2 \%{ }^{2}$.

Las primeras investigaciones acerca de esta patología, hablaban de una «disfunción cerebral mínima». Posteriormente, se hizo un mayor énfa-

Correspondencia a: Dr. Arturo Roizblatt S. Málaga 950, Depto. 52. Las Condes, Santiago, Chile.

E mail: ardasa@vtr.net sis en la hiperactividad, por lo que se acuñó el término de niño hiperquinético». Actualmente, se sabe que este síndrome puede o no acompañarse de hiperactividad ${ }^{3}$. Este tipo de trastorno puede persistir en la edad adulta desde $40 \%$ hasta $79 \%$ de los casos, por lo que se obtiene una prevalencia estimada de 3 a $6 \%$ en la población general ${ }^{4-6}$. La variabilidad de estos porcentajes se relaciona con los criterios diagnósticos empleados, quiénes fueron los informantes y los métodos que se consideraron para tomar la muestra de las diversas investigaciones.

Pese a su alta frecuencia, el TDAH es comúnmente subdiagnosticado o confundido con otras patologías psiquiátricas, lo cual retarda el tratamiento adecuado, deteriorando el mundo familiar, laboral y social de los pacientes. Es importante entonces, que el médico no especialista sea capaz de detectar esta patología, derivando al paciente 
en forma oportuna, sobre todo si consideramos que el no tratarlo puede llevar a consecuencias, sobre todo de la esfera psicosocial, difíciles de reparar. Por lo mismo, ante la presencia de un antecedente de déficit atencional de la infancia, debe ser norma averiguar por la persistencia de síntomas que hagan sospechar su persistencia en la adultez.

\section{Caso Cúnico}

Juan Carlos tiene 42 años, es separado en 2 oportunidades, con 3 hijos de ambos matrimonios, productor de televisión, con horarios de trabajo erráticos e inestabilidad laboral. Su actual pareja lo «obligó»a consultar, por ser dependiente de marihuana, consumo que minimizaba diciendo que «es sólo un pito por noche», lo que lo «calma» y le permite estar «más tranquilo». Nunca había sido tratado con fármacos ni psicoterapia.

En su biografía destacaba que fue abandonado por su madre a los 6 años (quien se fue a vivir al extranjero) siendo criado por su padre y sus 3 sucesivas madrastras.

Consideraba «normal»su dificultad para mantener la concentración, su necesidad de estar moviéndose constantemente, su elevado nivel de ansiedad e impulsividad. Refería que esas características «sólo me afectan en el área laboral»y le impidían desempeñar tareas que requerían concentración por tiempo prolongado, aunque posteriormente percibía que estas condiciones también han afectado sus relaciones interpersonales a nivel familiar y social.

Mencionaba el antecedente de déficit atencional con hiperactividad en su infancia, tratado con Ritalín ${ }^{\circledR}$ durante un período de varios años (que no precisaba con exactitud).

Podemos considerar que Juan Carlos tenía ciertas características que hacían pensar en un trastorno por déficit atencional del adulto, por lo que no es de sorprender la comorbilidad de su adicción y por otro lado su conflictiva familiar, frecuente de encontrar en este tipo de pacientes.

Fue tratado con bupropión (150 mg por día), psicoterapia individual y de pareja con lo cual mejoró su capacidad de concentración, disminuyó su hiperactividad, fue capaz de controlar en gran medida su impulsividad y sus relaciones interper- sonales mejoraron, vale decir mejoría tanto en el aspecto cognitivo como conductual, lo que fue de beneficio para él y el medio que lo rodeaba. Respecto al consumo de marihuana, ha disminuido bastante (expresa consumir sólo en fiestas y aproximadamente cada 15 días) y por razones que él califica como silosofía de vida»se ha negado a suspender.

Neuropatogenia. Se ha postulado que los neurotransmisores dopamina y norepinefrina estarían involucrados en funciones como la atención, concentración, motivación, interés y el aprendizaje de nuevas habilidades. Una disfunción de estos neurotransmisores estaría involucrada en la etiología del $\mathrm{TDAH}^{7}$, especialmente en áreas de la corteza prefrontal y de los ganglios basales ${ }^{8,9}$. Además, existiría una disminución global del consumo cerebral de glucosa, especialmente en la corteza premotora y corteza superior frontal, en los adultos con TDAH respecto a controles, lo que implicaría una disminución del metabolismo y de la actividad neuronal en esas zonas ${ }^{10}$.

Estos hallazgos tienen importancia para el tratamiento, ya que fármacos que aumentan los niveles de dopamina y norepinefrina -anfetaminas y bupropión entre otros- han demostrado ser eficaces en la disminución de los síntomas.

Con respecto a la genética de esta enfermedad, se postula un modelo de transmisión autosómico dominante. Sin embargo, sólo 51\% de los gemelos monocigotos estudiados presenta esta patología, por lo tanto, las influencias del ambiente también serían fundamentales ${ }^{8}$. Se ha comprobado además, que la exposición a tabaco de la madre durante el embarazo, se asocia a TDAH en los hijos ${ }^{11}$.

Clínica. El TDAH en el adulto se considera como la continuación de dicho trastorno en la infancia. Sus características son:

1. Hiperactividad motora: inquietud motora, incapacidad de relajarse, dificultad para persistir en actividades sedentarias (ej: ver televisión o leer el diario sin levantarse por un período de tiempo prudente). 2. Déficit de atención: incapacidad para concentrarse en una conversación o lectura, distractibilidad, olvidos frecuentes, constante pérdida de objetos (ej: billetera, llaves, olvidan reuniones y fechas importantes, durante una 
conversación saltan de un tema a otro, etc). 3. Labilidad emocional: cambios súbitos que van desde ánimo normal a depresión o euforia, llegando hasta excitación, generalmente de horas de duración. Esta característica genera inseguridad en las personas que los rodean ya que despierta una sensación de inseguridad en cuanto a la estabilidad afectiva. 4. Temperamento explosivo: episodios de pérdida de control de corta duración en que el sujeto puede temer por su propia conducta, lo que naturalmente interfiere en las relaciones interpersonales. 5. Hiperreactividad emocional: no puede soportar el estrés cotidiano y reacciona excesiva o inapropiadamente con desánimo, confusión, ansiedad o rabia, lo que impide el apropiado afrontamiento de los problemas, lo que lleva a las personas a tener dificultad en lo laboral y en sus relaciones afectivas. 6. Desorganización, incapacidad de completar tareas: poca capacidad para organizarse en el desarrollo del trabajo; las tareas por lo general quedan inconclusas, debido a que el sujeto se cambia constantemente de una actividad a otra, lo que proyecta una imagen que repercute en su evaluación. 7. Impulsividad: manifestaciones menores incluyen hablar antes de pensar, interrumpir las conversaciones de otros, impaciencia y otras acciones como compras compulsivas. Manifestaciones mayores son el abrupto inicio y término de relaciones (múltiples matrimonios, separaciones); comportamientos temerarios o imprudentes que implican riesgos no reconocidos como tales por el individuo (ej: dilapidaciones de dinero, conducción imprudente) ${ }^{12}$. 8. Condiciones asociadas al TDAH son inestabilidad matrimonial, menor éxito académico y laboral del esperado para su inteligencia y educación ${ }^{13}$, abuso de drogas y alcohol14,15, respuesta atípica a psicotrópicos, historia familiar de TDAH durante la infancia y trastorno de personalidad antisocial $^{12,16}$

Para poder configurar el diagnóstico, la Academia Americana de Psiquiatría (APA) en su Manual Diagnóstico y Estadístico de los Trastornos Mentales (DSM-IV-TR) requiere la presencia de una serie de síntomas que se agrupan en la Tabla 1.

Diagnóstico Diferencial. Las patologías que más frecuentemente pueden ser confundidas con TDAH en el adulto son los trastornos del ánimo y el trastorno de personalidad limítrofe.
A) Trastornos del ánimo: el TDAH del adulto puede ser confundido con trastornos del ánimo crónicos como los trastornos distímicos y ciclotímicos. A diferencia de estos cuadros, los síntomas del ánimo que se manifiestan en el TDAH son de corta duración, sin anhedonia (ausencia de placer al realizar actividades agradables) y sin síntomas físicos como insomnio, anorexia y baja de peso. Una emoción frecuentemente descrita por los pacientes es el «aburrimiento».

Los síntomas ciclotímicos del TDAH difieren de los síntomas del trastorno ciclotímico en que los períodos de ánimo elevado son de corta duración (horas), se caracterizan por excitación más que por euforia y son generalmente reactivos a situaciones del medio ambiente ${ }^{12}$.

B) Trastorno de personalidad limítrofe: los componentes del trastorno de personalidad limítrofe que se pueden ver en el TDAH incluyen impulsividad, ataques de ira, labilidad emocional y sentimientos de aburrimiento, sin embargo, la impulsividad de los pacientes con TDAH es de duración más breve, es situacional, moderada e intermitente, menos severa, además, pueden tener episodios de rabia en que explotan y se calman inmediatamente, por el contrario, los pacientes limítrofes persisten en sus sentimientos de rabia, y la demuestran con mayor intensidad.

Comorbilidad. La comorbilidad psiquiátrica es muy frecuente en los adultos con TDAH; afectaría a 3 de cada 4 pacientes y es muy importante buscarla ya que puede ser causa de mala respuesta al tratamiento. Las patologías con sus respectivas prevalencias se encuentran detalladas en la Tabla 2.

Tratamiento. Un tratamiento efectivo para el TDAH debe estar compuesto por 2 pilares: farmacológico y psicoterapéutico.

Los medicamentos que han demostrado una mayor efectividad en el control de los síntomas son los estimulantes: dextroanfetamina, metanfetamina, metilfenidato, y pemolina ${ }^{18-20}$. Aproximadamente $70 \%$ de los pacientes muestra mejoría cuando son tratados con metilfenidato $u$ otras anfetaminas ${ }^{21}$.

El metilfenidato (Ritalín ${ }^{\circledR}$ ) en adultos, ha mostrado una efectividad significativa al mejorar la capacidad de atención, reducir la impulsividad y 


\section{Tabla 1. C riterios D SM -IV-T R para T D AH tanto para niños como para adultos ${ }^{17}$}

A. (1) 0 (2):

(1) Seis o más de los siguientes síntomas de inatención han persistido por lo menos durante 6 meses con una intensidad que es desadaptativa e incoherente con el nivel de desarrollo:

\section{Inatención}

A menudo no presta atención suficiente a los detalles 0 incurren en errores por descuido en las tareas escolares, en el trabajo o en otras actividades.

A menudo tiene dificultades para mantener la atención en tareas 0 actividades lúdicas.

A menudo no sigue instrucciones y no finaliza tareas escolares, encargos u obligaciones en el centro de trabajo (no se debe a comportamiento negativista 0 a incapacidad para comprender instrucciones).

A menudo tiene dificultad para organizar tareas y actividades.

A menudo evita, le disgusta 0 es renuente en cuanto a dedicarse a tareas que requieren un esfuerzo mental sostenido (como trabajos escolares o domésticos).

A menudo extravía objetos necesarios para tareas 0 actividades (ej: libros, lápices, etc.)

A menudo se distrae fácilmente por estímulos irrelevantes.

A menudo es descuidado en las actividades diarias.
(2) Seis o más de los siguientes síntomas de hiperactividad-impulsividad han persistido por lo menos durante 6 meses con una intensidad que es desadaptativa e incoherente en relación con el nivel de desarrollo:

\section{Hiperactividad}

A menudo mueve en exceso manos o pies, o se remueve en su asiento.

A menudo abandona su asiento en la clase $u$ otras situaciones en que se requiera estar sentado.

A menudo corre o salta excesivamente en situaciones en que es inapropiado hacerlo (en adultos puede limitarse a sentimientos subjetivos de inquietud).

A menudo tiene dificultades para jugar 0 dedicarse tranquilamente a actividades de ocio.

A menudo «está en marcha»0 suele actuar «como si tuviera un motor».

A menudo habla en exceso.

\section{Impulsividad}

A menudo precipita respuestas antes de haber sido completadas las preguntas.

A menudo tiene dificultades para esperar su turno.

A menudo interrumpe o se inmiscuye en las actividades de otros (ej: se inmiscuye en las conversaciones de otros).

B. Algunos síntomas de hiperactividad-impulsividad o inatención que causaban alteraciones estaban presentes antes de los 7 años de edad.

C. Algunas de las alteraciones provocadas por los síntomas se presentan en 20 más ambientes (ej: en el trabajo 0 escuela y en la casa).

D. Deben existir pruebas claras de un deterioro clínicamente significativo de la actividad social, académica o laboral.

E. Los síntomas no aparecen exclusivamente en el transcurso generalizado del desarrollo, esquizofrenia u otro trastorno psicótico, y no se explican mejor por la presencia de otro trastorno mental (ej: trastorno del estado de ánimo, trastorno de ansiedad, trastorno disociativo, o trastorno de la personalidad).

Especificar según tipo:

F90.0 Trastorno por déficit atencional con hiperactividad, tipo combinado: si se satisfacen los criterios A(1) y A(2) durante los últimos 6 meses.

F90.8 Trastorno por déficit atencional con hiperactividad, con predominio de déficit de la atención: si se satisface el criterio $\mathrm{A}(1)$, pero no el $\mathrm{A}(2)$ durante los últimos 6 meses.

F90.0 Trastorno por déficit atencional con hiperactividad, tipo con predominio hiperactivo-impulsivo: si se satisface el criterio $\mathrm{A}(2)$, pero no el $\mathrm{A}(1)$ durante los últimos 6 meses.

* Nota de codificación. En el caso de sujetos (en especial adolescentes y adultos) que actualmente presenten los síntomas, pero que ya no cumplen todos los criterios, debe especificarse «en remisión parcial» 
Tabla 2. Comorbilidad psiquiátrica en pacientes adultos con T D AH ${ }^{18}$

\begin{tabular}{|lc|}
\hline Tipo de Trastorno & Frecuencia observada \\
\hline * Abuso y dependencia de alcohol & $32-53 \%$ \\
* Trastornos por ansiedad & $25-50 \%$ \\
* Trastornos del ánimo & $20-40 \%$ \\
* Conductas antisociales & $18-38 \%$ \\
* Alteraciones del aprendizaje (ej: dislexia) & $20 \%$ \\
* Abuso y dependencia de sustancias (marihuana, cocaína, etc.) & $8-32 \%$ \\
* Trastornos de personalidad en general & $10-20 \%$ \\
\hline
\end{tabular}

disminuir notoriamente la hiperactividad moto$\mathrm{ra}^{22}$, aunque su uso ha sido cuestionado por sus efectos secundarios y riesgo de dependencia 23 , distintas investigaciones han confirmado su indicación en este trastorno 24 . Incluso pacientes que presentaban comorbilidad con abuso de drogas 0 dependencia a cocaína en los cuales la adherencia al tratamiento es menor, mostraron mejoría ${ }^{25}$. Para iniciar el tratamiento, se recomienda una dosis de $5 \mathrm{mg}$ al desayuno (se debe ingerir con las comidas ya que mejora su absorción), y luego ir aumentando cada 3 ó 4 días según la respuesta del paciente, hasta alcanzar una dosis máxima de $60 \mathrm{mg} /$ día, dividida en 2-3 dosis de 20 ó $30 \mathrm{mg}^{26}$, aunque debiera ser indicado sólo por especialistas.

Las anfetaminas están contraindicadas en pacientes psicóticos, epilépticos (bajan el umbral convulsivo), con disfunción hepática, que presenten tics, glaucomatosos, y aquellos que estén usando antidepresivos IMAO (inhibidores de la monoaminoxidasa). Los efectos secundarios al uso de metilfenidato en adultos son similares a los que presentan los niños, siendo los más frecuentes la disminución del apetito y el insomnio. Baja de peso, cefalea, náuseas, trastornos gastrointestinales y disforia se han descrito en forma excepcional ${ }^{27}$.

Los antidepresivos también han demostrado ser eficaces en el tratamiento de esta patología, con la ventaja adicional de cubrir la comorbilidad depresiva. Según el tipo de antidepresivo, pueden aumentar los niveles de dopamina y/o norepinefrina. Es importante recalcar que pese a ser efectivos, sus resultados son más modestos que con las anfetaminas. El bupropión es un antidepresivo dopaminérgico y noradrenérgico, está indicado en dosis diarias de 150 a 300 $\mathrm{mg}^{28}$. Los antidepresivos tricíclicos (amitriptilina, imipramina), se sugieren en dosis tan bajas como $10 \mathrm{mg}$ o $25 \mathrm{mg}$ por día. Si no hay respuesta, se pueden incrementar hasta alcanzar los 150-300 mg al día ${ }^{29}$. También se han usado antidepresivos IMAO, con una eficacia menor y se reservan para aquellos pacientes refractarios a otros medicamentos. Los inhibidores de la recaptación de serotonina (fluoxetina, paroxetina, sertralina, citalopram), estarían indicados en TDAH cuando -además de un trastorno afectivohay comorbilidad con ansiedad u obsesivi$\operatorname{dad}^{18,28}$.

La atomoxetina, un potente inhibidor presináptico del transportador de norepinefrina, ha sido recientemente aprobado por la Food and Drug Administration de los Estados Unidos de Norteamérica (FDA) para el tratamiento de esta patología. Si bien existen pocos estudios en adultos, éstos demuestran que es superior a placebo en dosis de 76 a $90 \mathrm{mg} /$ día, diferenciándose de las anfetaminas en que no produciría dependencia ${ }^{30,31}$. Sus efectos adversos más comunes son cefalea, disminución del apetito, sequedad bucal, náuseas y vómitos ${ }^{32,33}$. Aún no está disponible en nuestro país.

En relación a la psicoterapia, las técnicas cognitivo-conductuales y la terapia sistémica tendrían efectos en la baja autoestima, en el control de la impulsividad, en el aprendizaje de destrezas para resolver problemas y disponer de nuevos repertorios para enfrentar conflictos en las relaciones interpersonales ${ }^{34,35}$, mejorando la calidad de vida del paciente tanto familiar como laboral. El tratamiento en conjunto de terapia conductual y metilfenidato es más efectivo que cada uno por separado ${ }^{26}$. También será importante considerar 
una adecuada elección de la actividad laboral, la que debe ser compatible con las características que son propias de esta enfermedad.

En conclusión el TDAH del adulto es una entidad clínica frecuente; sin embargo, es subdiagnosticada y, por lo tanto, subtratada. Las consecuencias para la persona que lo sufre y su entorno pueden ser desastrosas (conflictos matrimoniales, laborales, accidentes). De este modo, el TDAH debe ser considerado en la práctica médica general, teniendo presente los criterios aportados por la American Psychiatric Association en el DSM-IVTR. Una vez identificado el trastorno, debe ser derivado al médico especialista para su correcto

\section{REFERENCIAS}

1. Faraone S, Biederman J, Mick E, Wimamson S, Wilens T, Spencer T et al. Family study of girls with attention deficit hyperactivity disorder. Am J Psychiatry 2000; 157: 1077-83.

2. Toledo V, De la Barra F, López C, George M, RodRíguez J. Diagnósticos psiquiátricos en una cohorte de escolares de primer año de enseñanza básica del Área Occidente de Santiago. Rev Chil Neuro-Psiquiat 1997; 35: 17-24.

3. Clements SED, Peters JE. Minimal brain disfunction in the school age child. Arch Gen Psychiatry 1962; 6: 185-97.

4. Barkiey RA, Fisher M, Edelbrock CS, Smawish L. The adolescent outcome of hyperactive children diagnosed by research criteria. An 8 years prospective follow up study. J Am Acad Child Adolesc Psychiatry 1990; 29: 546-57.

5. SCHAFFER D. Attention deficit hyperactivity disorder in adults. Am J Psychiatry 1994; 151: 633-8.

6. Wender PH. Attention-Deficit Hyperactivity Disorder. Psychiatr Clin North Am 1998; 21: 761-74.

7. Stahl SM. Cognitive Enhancers. En: Stahl SM. Essential Psychopharmacology. Cambridge: Cambridge University Press, 2000; 459-67.

8. ZametKin AJ, Liotta W. The Neurobiology of Attention-Deficit/Hyperactivity Disorder. J Clin Psychiatry 1998; 59 (suppl 7): 17-23.

9. ERnst M, Zametrin AJ, Matochik JA, Jons PH, Cohen RM. DOPA Decarboxylase Activity in Attention Deficit Hyperactivity Disorder in Adults. A [Fluorine-18] Fluorodopa Positron Emission Tomographic Study. J Neurosci 1998; 18: 5901-7. tratamiento. Este debe estar basado en la farmacoterapia, principalmente a través de estimulantes para mejorar la atención, la concentración y la hiperactividad si es que existe. También debe realizarse un tratamiento de psicoterapia cognitivo-conductual y sistémica (sea individual, matrimonial o familiar) que ayuden al paciente y a su entorno a desenvolverse en forma adecuada en su vida cotidiana, evitando que sintomatología no tratada afecte la vida laboral, familiar y social. Estamos seguros que es el médico general quien más puede colaborar en la pesquisa precoz y por lo mismo derivación oportuna de estos pacientes.

10. ZametKin AJ, Nordahl TE, Gross M, King AC, Semple WE, RUMSEY J ET AL. Cerebral glucose metabolism in adults with hyperactivity of childhood onset. $\mathrm{N}$ Engl J Med 1990; 323: 1361-6.

11. Linnet KM, Dalsgaard S, Obel C, Linnet KM, Dalsgaard S, OBel C et al. Maternal lifestyle factors in pregnancy risk of attention deficit hyperactivity disorder and associated behaviors: review of the current evidence. Am J Psychiatry 2003; 160: 1028-40.

12. WendER P. Adult Manifestations of Attention Deficit/Hyperactivity Disorder. En: Kaplan H, Sadock B, editores. Comprehensive Textbook of Psychiatry. Philadelphia: Lippincot, Williams and Wilkins, 2000; 2688-91.

13. HinshaW SP. Academic underachievement, attention deficits, and aggression: Comorbidity and implications for intervention. J Consult Clin Psychol 1992; 60: 893-903.

14. KieIN RG. Prognosis of attention deficit disorder in management in adolescence. Pediatr Rev 1987; 8: 216-22.

15. LAMBERT NM. Adolescent outcomes of hyperactive children. Perspectives on general and specific patterns of childhood risk for adolescent emotional, social and mental health problems. J Am Psychol 1988; 43: 786-99.

16. MorRISON JR. Childhood hyperactivity in an adult psychiatric population. Social factor. J Clin Psychiatry 1980; 41: 40-3.

17. American Psychiatric Association. Manual Diagnóstico y Estadístico de Trastornos Mentales, cuarta edición, texto revisado. Barcelona: Editorial Masson, 2002; 97-107. 
18. Wilens TE, Spencer TJ, Biederman J. Pharmacotherapy of adult ADHD. En K.G. Nadeau Ed. A comprehensive guide to attention deficit disorder in adults: Research, diagnosis and treatment. New York: Brunner/Mazel, 1995; 168-88.

19. Wilens TE, Biederman J, Spencer TJ, Prince J. Pharmacotherapy of adult attention deficit/hyperactivity disorder: A review. J Clin Psychopharmacol 1995; 15: 270-9.

20. Elia J, Ambrosini PJ, Rapoport JL. Treatment of Attention-Deficit-Hyperactivity Disorder. N Engl J Med 1999; 340: 780-8.

21. Ешот H. Attention Deficit Hyperactivity Disorder in Adults: A Guide for the Primary Care Physician. South Med J 2002; 95: 736-42.

22. Wender PH, ReImHerr F, Wood D, WARD MA. Control study of metilphenidate in the treatment of attention deficit disorder, residual type, in adults. Am J Psychiatry 1985; 142: 547-52.

23. Gawin FH, Ruordan C, Kleber HD. Metilphenydate treatment of cocaine abusers without attention deficit disorder. A negative report. Am J Drug Alcohol Abuse 1985; 11: 193-7.

24. Spencer T, Wilens T, Biederman J, Faraone SV, Ablon JS, LAPEY K. A double blind, crossover comparison of metilphenidate and placebo in adults with childhood onset of attention-deficit hyperactivity disorder. Arch Gen Psychiatry 1995; 52: 434-43.

25. Khantzian EJ, Gawin F, Kleber HD, Riordan CE. Methylphenidate (ritalin) treatment of cocaine dependence: Preliminary report. J Subst Abuse Treat 1984; 1: 107-12.
26. Elia J, Ambrosini P, Rapoport JL. Treatment of Attention Deficit-Hyperactivity Disorder. N Engl J Med 1999; 340: 781-8.

27. Wender PH, ReimherR FW, Wood DR. Attention DEFICIT DISORDER, MINIMAL BRAIN DAMAGE in adults. A replication study of diagnosis and drug treatment. Arch Gen Psychiatry 1981; 38: 449-56.

28. Puiszka SR. The serotonin re-uptake blockers; Do they have a role in the treatment of ADHD? The ADHD report 1995; 3: 3-5.

29. Wilens TE, Biederman J, SPENCER TJ. Farmacoterapia del Síndrome de Déficit Atencional e Hiperactividad en el Adulto. CNS Drugs 1998; 9: 347-56.

30. Spencer T, Biederman J, Wilens T, Prince J, Hatch M, Jones J ET AL. Am J Psychiatry 1998; 155: 693-5.

31. Michelson D, Adier L, Spencer T, Reimherr F, West S, Auen A ET AL. Atomoxetine in Adults with ADHD: Two Randomized Placebo-Controlled Studies. Biol Psychiatry 2003; 53: 112-20.

32. Buck M. New Agents and Second-line Therapies for Attention-Deficit/Hyperactivity Disorder. Pediatr Pharm 2002; 8: 11-16.

33. Michelson D, Aluen AJ, Busner J, Casat C, Dunn D, KRATOChVIL C ET AL. Once daily atomoxetine treatment for children and adolescents with Attention Deficit Hyperactivity Disorder: a randomized, placebo controlled study. Am J Psychiatry 2002; 159: 1896-901.

34. Weinstein C. Cognitive remediation strategies. J Psychother Pract Res 1994; 3: 44-57.

35. Halowell EM, Ratey JJ. Driven to destruction. New York: Pantheon Books, 1994: 30-5. 